\title{
Importância da avaliação e padronização analgésica em serviços de emergência*
}

\author{
Importance of pain evaluation and standardization of analgesic medication in \\ emergency services
}

Importancia de la evaluación y estandartización analgésica en servicios de emergencia

\author{
Ana Marial Calil ${ }^{1}$, Cibele Andrucioli de Mattos Pimenta ${ }^{2}$
}

\begin{abstract}
RESUMO
Objetivos: Conhecer a medicação antiálgica utilizada em pacientes que sofreram acidentes de transporte, o padrão analgésico e pontuar a intensidade da dor. Métodos:Estudo descritivo, exploratório e longitudinal com abordagem quantitativa, que abrangeu 100 pacientes envolvidos em acidentes de transporte admitidos para tratamento em hospital referência para trauma. Resultados:A dor foi identificada em 90,0\% dos pacientes, sendo as de forte e média intensidade as mais frequentes; não se encontrou prescrição antiálgica em $48 \%$ dos casos. Maior uso medicamentoso foi encontrado em vítimas com lesões em membros e nos sete casos em que a dor foi totalmente aliviada, $5(66,7 \%)$ foram utilizados opióides. Conclusão: A dor é um fenômeno comum associado ao trauma com sub-tratamento e sub-avaliação em nosso meio, e o uso de opióides para dores reconhecidamente muito intensas ainda é um recurso pouco utilizado na emergência, mesmo em pacientes hemodinamicamente estáveis e com Escala de Coma de Gasglow=15.
\end{abstract}

Descritores: Dor; Ferimentos e lesões; Analgesia; Serviços médicos de emergência; Avaliação.

\begin{abstract}
Objectives: To describe the types of analgesic medication used by patients who had automobile accidents and to identify standardization of analgesic medication and measurement of pain intensity. Methods: This was a descriptive longitudinal quantitative study with 100 patients who had automobile accidents from a trauma designated hospital. Results: The majority of patients (90.0\%) reported having pain. High and moderate levels of pain intensity were the most commonly reported by the patients. There was no prescription of analgesic medication for $48 \%$ of the patients. Patients with limb lesions received more analgesic medication than those with other types of lesions. Among 7 patients who had complete pain relief, 5 of them (66.7\%) received opioid medication. Conclusions: Pain is a common symptom associated with inadequate evaluation and management. The use of opioid medication to relief high intensity pain in trauma patients in emergency services is not a common practice even for those patients who are hemodynamically stable and having a score of 15 on the Glasgow Coma Scale.
\end{abstract}

Key Words: Pain, Wounds and Lesions; Analgesia; Medical Emergency Services; Evaluation.

\section{RESUMEN}

Objetivos: Conocer los medicamentos antiálgicos utilizados en pacientes que sufrieron accidentes de transporte, conocer el estándar analgésico y dar puntajes a la intensidad del dolor. Métodos: Estudio descriptivo, exploratorio y longitudinal con abordaje cuantitativo, que comprendió 100 pacientes que sufrieron accidentes de transporte y fueron admitidos para tratamiento en un hospital de referencia para tratamiento de traumas. Resultados: El dolor fue identificado en 90,0\% de los pacientes, siendo los dolores más frecuentes los de fuerte y media intensidad; no se encontró prescripción antiálgica en 48\% de los casos. El mayor uso medicamentoso fue encontrado en víctimas con lesiones en miembros y entre los siete casos en que el dolor fue totalmente aliviado, en 5 (66,7\%) fueron utilizados opiáceos. Conclusión: El dolor es un fenómeno común asociado al trauma con sub-tratamiento y sub-evaluación en nuestro medio, y el uso de opiáceos para dolores reconocidamente muy intensos también es un recurso poco utilizado en emergencias, inclusive en pacientes hemodinámicamente estables y con Escala de Coma de Gasglow=15.

Palabras clave: Dolor; Heridas y lesiones; Analgesia; Servicios médicos de emergencia; Evaluación.

\footnotetext{
* Parte da tese de doutorado intitulada: "Dor e analgesia em vítimas de acidentes de transporte atendidas em um pronto-socorro", apresentada à Escola de Enfermagem da Universidade de São Paulo. São Paulo (SP), Brasil. Vencedor do prêmio Simbidor-2007 de melhor pesquisa na área de tratamento da dor.

1 Doutora em Enfermagem. Professora e colaboradora do Curso de Especialização em Emergência do Centro Universitário São Camilo. São Paulo (SP), Brasil.

${ }^{2}$ Professora Titular do Departamento de Enfermagem Médico Cirúrgica da Escola de Enfermagem da Universidade de São Paulo. São Paulo (SP), Brasil.
} 


\section{INTRODUÇÃO}

Dor é uma das principais conseqüências do trauma e suas repercussões são potencialmente prejudiciais ao organismo ${ }^{(1)}$. Embora freqüente, pouca atenção tem sido concedida ao controle álgico do traumatizado e o tema é insuficientemente pesquisado em nosso meio ${ }^{(2)}$.

As repercussões orgânicas do processo álgico intenso são subestimadas por médicos e enfermeiros; a desinformação sobre as técnicas disponíveis e sobre a farmacologia das drogas analgésicas; condições do local do acidente e do setor de emergência adversas a esse tipo de tratamento e a administração precoce de analgésicos podendo mascarar um indício valioso para o diagnóstico etiológico são os principais motivos citados na literatura para o inadequado controle da dor no setor de emergência ${ }^{(2-3)}$.

Dor é uma experiência sensorial e emocional desagradável, associada a uma lesão tissular real ou potencial, e descrita em termos de tal dano ${ }^{(4)}$. A dor aguda está relacionada a afecções mecânicas, químicas e térmicas, como aquelas advindas de traumas, queimaduras, infecções e processos inflamatórios ${ }^{(4)}$.

A dor aguda pode levar a respostas como a elevação da pressão arterial, o aumento das frequências cardíaca e respiratória, entre outras, que pode resultar em hipoventilação, aumento do trabalho cardíaco e diminuição da perfusão sangüínea periférica. Nos quadros hemorrágicos, os estímulos nociceptivos podem agravar o choque, pelo aumento do trabalho do ventrículo esquerdo e redução da oferta de oxigênio $^{(4-5)}$. O controle da dor, além de humanitário, é vital para a assistência imediata ao acidentado, visando manter as funções fisiológicas básicas e evitar efeitos deletérios.

No setor de emergência grande parte dos atendimentos é decorrente de causas externas, constituídas por acidentes e violências, conforme Classificação Estatística Internacional de Doenças e Problemas Relacionados à Saúde (CID-10) ${ }^{(6)}$.

O perfil epidemiológico brasileiro associado a carência de literatura nacional que aborde esse tema é preocupante, visto o grande número de vítimas que são atendidas e permanecem diariamente nesse setor. Além disso, não existe nos manuais de atendimento às vítimas de trauma um capítulo específico dedicado ao tema dor, analgesia e a utilização de instrumentos de avaliação para aferir o quadro álgico.

Acredita-se fundamental o conhecimento de farmacologia, técnicas analgésicas e possibilidades de avaliação da dor por meio de instrumentos objetivos, úteis e viáveis no cenário da emergência. Desse modo, estabeleceu-se como objetivos desse estudo: conhecer a medicação antiálgica utilizada em pacientes que sofreram acidentes de transporte, o padrão analgésico e pontuar a intensidade da dor.

\section{MÉTODOS}

Trata-se de estudo descritivo, exploratório e longitudinal, realizado por meio documental (prontuário) e pesquisa de campo. Foi desenvolvido no Pronto-Socorro Cirúrgico (PSC) de um hospital geral governamental, centro de referência para o atendimento ao trauma, no Município de São Paulo. A coleta de dados foi iniciada após a aprovação do projeto de pesquisa pelo Comitê de Pesquisa e Ética do referido hospital, parecer número: 074/02.

Foram avaliadas todas as vítimas de acidentes de transporte que deram entrada no referido serviço nos meses de maio e junho no ano de 2002. Se a entrevistadora estivesse em campo, a avaliação era iniciada imediatamente após o primeiro atendimento. Se a entrevistadora não estivesse em campo era acionada a comparecer no hospital de coleta. Durante o período proposto, foram avaliadas 100 vítimas que atenderam aos seguintes critérios de inclusão: provenientes diretamente da cena do evento; escore igual ou superior a 15 na Escala de Coma de Glasgow (ECGl), não medicados antes da entrada no PSC e idade igual ou superior a 16 anos. Garantiu-se que os pacientes estivessem conscientes e orientados (por meio da ECGl e controle de parâmetros vitais), por ser fundamental para a precisão da avaliação da intensidade dolorosa.

As fontes de dados utilizadas foram o livro de registro de entrada de doentes, a ficha de admissão no PSC, o prontuário, o exame físico e a entrevista. Por meio do livro de registro, a pesquisadora localizou os pacientes no setor de PSC e os convidou a participar da pesquisa, após assinatura do Termo de Consentimento Livre e Esclarecido, o mesmo ocorrendo com os responsáveis pelos menores.

Para cada paciente foi aberta uma ficha de coleta, construída visando caracterizar a dor e os analgésicos utilizados. Estabeleceu-se que o exame físico e a entrevista seriam realizados após a avaliação primária e secundária e a estabilização do quadro. Utilizou-se o New Injury Severity Score (NISS) para aferir a gravidade do trauma, medido por meio do somatório das três regiões corpóreas mais gravemente atingidas; NISS maior ou igual a 16 é indicativo de trauma grave.

A intensidade da dor foi avaliada pela escala analógica visual, em dois momentos, e a localização da dor mais intensa pelo diagrama corporal. A intensidade dolorosa foi categorizada em: 0 - ausência de dor; 1 a 4 dor leve; 5 a 7 dor moderada e 8 a 10 dor intensa

A descrição abaixo demonstra as situações de avaliação:

- Pacientes avaliados na presença da pesquisadora no PSC, primeira pergunta foi realizada após a avaliação inicial do Advanced Trauma Life Support (ATLS) e a segunda pergunta após uma hora da intervenção analgésica ou após três horas sem intervenção analgésica.

- Pacientes no PSC (sem tratamento analgésico), primeira pergunta: na chegada da pesquisadora e a segunda pergunta após uma hora da intervenção analgésica ou após três horas sem intervenção analgésica.

- Pacientes no PSC (com tratamento analgésico), primeira pergunta: na chegada da pesquisadora e a segunda pergunta após uma hora da intervenção analgésica ou após três horas sem intervenção analgésica.

O período de uma hora foi adotado como segurança para o início e tempo médio de ação medicamentosa (Dor I). O período de três horas foi o tempo máximo esperado (Dor II) para a 
instalação de terapêutica antiálgica e a viabilidade do estudo.

As intervenções medicamentosas analgésicas foram investigadas no prontuário do paciente, e todas puderam ser resgatadas, por meio da observação da checagem no prontuário, da administração, e o controle, pela farmácia, da liberação de medicamentos, com especial cuidado para opióides e outros psicotrópicos.

As prescrições analgésicas foram categorizadas em três grupos, de acordo com os analgésicos encontrados: analgésicos simples (dipirona e paracetamol); antiinflama-tório nãohormonal (diclofenaco/cetoprofeno/tenoxican) e opióides (tramadol/meperidina/codeína/morfina/fenta-nila).

Orientação estatística garantiu que o número de 100 pacientes coletados durante os dois meses seriam suficientes para uma amostra representativa da população de origem e para as análises propostas. Os dados foram lançados em base de dados para o processamento das análises descritivas e por inferências. Os resultados foram organizados em tabelas e quadros e as freqüências em números absolutos e relativos

O teste não paramétrico de Mann-Whitney foi utilizado para verificar a comparação de dois grupos indepen-dentes em relação a uma variável quantitativa, devido as variáveis não apresentarem distribuição normal (suposição de normalidade dos dados rejeitada) $)^{(7)}$.

Já o teste de McNemar, foi utilizado para avaliar a existência de relação entre as proporções, em dois momentos de avaliação distintos ${ }^{(7)}$.

Todos os testes foram realizados admitindo-se nível de significância de 5\%.

\section{RESULTADOS}

Observou-se, na primeira avaliação, que $56,0 \%$ dos pacientes apresentaram dor intensa e $29,0 \%$ dor moderada. $\mathrm{Na}$ segunda avaliação, 26,0\% referiram dor intensa, 38,0\% dor moderada, e apenas $7,0 \%$ tiveram sua dor totalmente alivia$\mathrm{da}$, visto que na primeira avaliação 10 vítimas referiram não sentir dor e na segunda, 17 vítimas. As 10 vítimas que referiram não sentir dor na primeira avaliação confirma-ram a ausência da mesma na segunda avaliação (Tabela 1).

Os pacientes referiram melhora da intensidade dolorosa entre os dois momentos. Testes estatísticos para comparação da avaliação dos níveis de dor (todos ao mesmo tempo) não puderam ser realizados. Indicou-se a possibilidade de comparação por meio de dois grupos: sem dor + dor leve e dor moderada + dor intensa. Foi realizada, para cada vítima, uma trajetória da pontuação da própria dor (denominada de evolução da dor). Os resultados da primeira avaliação e da segunda, assim como Teste de McNemar permitindo observar alte-ração significativa entre os dois momentos de avaliação, em relação à intensidade dolorosa $(p=0,001)$.

A Tabela 2 mostra que das 48 vítimas que não receberam analgesia, 43 permaneceram com a mesma inten-sidade dolorosa, ou seja, sem nenhuma melhora na escala analógica visual. Em relação aqueles que receberam medi-cação antiálgica, $10(23,8 \%)$ não apresentaram nenhum grau de melhora da dor e os demais apresentaram graus de melhora variados. O teste de Mann Whitney mostrou existir diferença estatistica-mente significativa na melhora entre os dois grupos $(p=0,001)$.

Um total de 18 pacientes receberam medicação na sala de emergência e durante o período de observação. Observa-se que em 16 pacientes o mesmo fármaco prescrito no setor de emergência foi utilizado em um momento posterior, durante o período de observação. Em um caso houve alteração de um antiinflamatório não hormonal (AINH) por um opióide

Tabela 1. Intensidade dolorosa em pacientes vítimas de acidente de transporte em dois momentos. São Paulo, maio e junho de 2002.

\begin{tabular}{lcccc}
\hline \multirow{2}{*}{ Avaliação da dor } & \multicolumn{2}{c}{ Primeir a Avaliação } & \multicolumn{2}{c}{ Segunda Avaliação } \\
\cline { 2 - 5 } & $\mathbf{n}$ & $\mathbf{0}$ & $\mathbf{n}$ & $\mathbf{0}$ \\
\hline Ausência de dor & 10 & 10,0 & 17 & 17,0 \\
Dor leve & 5 & 5,0 & 19 & 19,0 \\
Dor moderada & 29 & 29,0 & 38 & 38,0 \\
Dor intensa & 56 & 56,0 & 26 & 26,0 \\
\hline Total & $\mathbf{1 0 0}$ & $\mathbf{1 0 0 , 0}$ & $\mathbf{1 0 0}$ & $\mathbf{1 0 0 , 0}$ \\
\hline
\end{tabular}

Tabela 2. Pacientes vítimas de acidente de transporte com dor ( $\mathrm{n}=90)$, medicados e não medicados, segundo pontos de melhora na escala analógica visual (EAV). São Paulo, maio e junho de 2002.

\begin{tabular}{lcccccc}
\hline \multirow{2}{*}{ Escala de melhor a } & \multicolumn{2}{c}{ Não medicados } & \multicolumn{2}{c}{ Medicados } & \multicolumn{2}{c}{ Total } \\
\cline { 2 - 7 } & $\mathbf{n}$ & $\mathbf{\%}$ & $\mathbf{n}$ & $\mathbf{\%}$ & $\mathbf{n}$ & $\%$ \\
\hline 0,00 & 43 & 89,6 & 10 & 23,8 & 53 & 58,9 \\
1,00 & 1 & 2,1 & 1 & 2,4 & 2 & 2,2 \\
2,00 & 1 & 2,1 & 4 & 9,5 & 5 & 5,6 \\
3,00 & - & - & 3 & 7,1 & 3 & 3,3 \\
4,00 & - & - & 8 & 19,0 & 8 & 8,9 \\
5,00 & 1 & 2,1 & 5 & 11,9 & 6 & 6,7 \\
6,00 & 1 & 2,1 & 2 & 4,8 & 3 & 3,3 \\
7,00 & - & - & 2 & 4,8 & 2 & 2,2 \\
8,00 & 1 & 2,1 & 5 & 11,9 & 6 & 6,7 \\
10,00 & - & - & 2 & 4,8 & 2 & 2,2 \\
\hline Tot al & 48 & 53,3 & 42 & 46,7 & 90 & 100,0 \\
\hline
\end{tabular}

Mann Whitney $=6,09 ; p=0,001-$ não houveponto de melhora $=9,00$ 
Quadro 1. Medicações utilizadas no atendimento inicial e no período de observação de pacientes vítimas de acidentes de transporte. São Paulo, maio e junho de 2002.

\begin{tabular}{|c|c|c|c|}
\hline $\begin{array}{c}\text { Dor I - Dor II } \\
\text { (EAV) }\end{array}$ & NISS & Setor de Emergência & Período de observação \\
\hline $8-6$ & 9 & Dipirona $-3 \mathrm{ml} I V$, dose única & Dipirona $-3 \mathrm{ml} I V$, dose única \\
\hline $10-0$ & 17 & Profenid - 50mg IV - dose única & Tramadol 50mg IV -6/6h \\
\hline $10-6$ & 5 & $\begin{array}{l}\text { Meperidina - sol. decimal } 2 \mathrm{ml} \mathrm{IV} \text {, } \\
\text { dose única }\end{array}$ & $\begin{array}{l}\text { Meperidina - sol. decimal } 2 \mathrm{ml} \mathrm{IV} \text {, dose } \\
\text { única }\end{array}$ \\
\hline $8-0$ & 17 & Tramadol - 50mg IV, dose única & Tramadol - 50mg IV, dose única \\
\hline $5-0$ & 5 & Tramadol - 50mg IV, dose única & Tramadol - 50mg IV, dose única \\
\hline $10-3$ & 17 & $\begin{array}{l}\text { Meperidina - sol. decimal } 2 \mathrm{ml} \mathrm{IV} \text {, } \\
\text { dose única }\end{array}$ & Tramadol - 50mg- IV , 8/8h \\
\hline $8-0$ & 13 & $\begin{array}{l}\text { Meperidina - sol. decimal } 2 \mathrm{ml} \mathrm{IV} \text {, } \\
\text { dose única }\end{array}$ & Tramadol $-50 \mathrm{mg}-\mathrm{IV}, 8 / 8 \mathrm{~h}$ \\
\hline $10-7$ & 12 & $\begin{array}{l}\text { Meperidina - sol. decimal } 2 \mathrm{ml} \mathrm{IV} \text {, } \\
\text { dose única }\end{array}$ & $\begin{array}{l}\text { Meperidina - sol. decimal 2ml IV, dose } \\
\text { única }\end{array}$ \\
\hline $8-2$ & 5 & Morfina - 5mg IV, dose única & Morfina - 5mg IV, dose única \\
\hline $10-6$ & 5 & $\begin{array}{l}\text { Meperidina - sol. decimal } 2 \mathrm{ml} \mathrm{IV} \text {, } \\
\text { dose única }\end{array}$ & $\begin{array}{l}\text { Meperidina - sol. decimal } 2 \mathrm{ml} \mathrm{IV,} \mathrm{dose} \\
\text { única }\end{array}$ \\
\hline $7-7$ & 17 & Dipirona -35 gts, dose única & Dipirona -35 gts, dose ú nica \\
\hline $10-0$ & 2 & Diclofenaco - IM, dose única & Diclofenaco-IM 8/8h \\
\hline $5-3$ & 2 & Dipirona $-2 \mathrm{ml} \mathrm{IV}$, dose única & Dipirona $-2 \mathrm{ml} \mathrm{IV}$, dose única \\
\hline $10-2$ & 6 & Diclofenaco - IM, dose única & Diclofenaco - IM, dose única \\
\hline $5-5$ & 9 & Dipirona $-3 \mathrm{ml} I V$, dose única & Dipirona $-3 \mathrm{ml} \mathrm{IV}$, dose única \\
\hline $8-0$ & 5 & Dipirona $-3 \mathrm{ml} I V$, dose única & Dipirona $-3 \mathrm{ml} \mathrm{IV}$, dose única \\
\hline $7-2$ & 6 & Dipirona $-3 \mathrm{ml} I V$, dose única & Dipirona $-3 \mathrm{ml} \mathrm{IV}$, dose única \\
\hline $10-6$ & 4 & Dipirona - 30 gts, dose única & Dipirona - 30 gts, dose ú nica \\
\hline
\end{tabular}

$E A V=E A V-$ Escala An alógica Visual; NISS = New Injury Severity Score.

Quadro 2. Medicações utilizadas apenas na sala de emergência para pacientes vítimas de acidentes de transporte. São Paulo, maio e junho de 2002.

\begin{tabular}{|cc|l|}
\hline $\begin{array}{c}\text { Dor I - Dor II } \\
\text { EAV }\end{array}$ & NISS & Setor de Emergência \\
\hline $10-2$ & 5 & Morfina $-4 \mathrm{mg}$ IV, dose única \\
\hline $8-6$ & 3 & Dipirona $-2 \mathrm{ml}$ IV, dose única \\
\hline $10-6$ & 9 & Meperidina - sol. decimal $2 \mathrm{ml}$ IV, dose única \\
\hline $6-6$ & 2 & Dipirona $-2 \mathrm{ml}$ IV, dose única \\
\hline $10-10$ & 17 & Morfina $-4 \mathrm{mg}$ IV, dose única \\
\hline $7-7$ & 11 & Dipirona $-2 \mathrm{ml}$ IV, dose única \\
\hline $10-6$ & 4 & Diclofenaco - IM, dose única \\
\hline
\end{tabular}

EAV-Escala An alógica Visual; NISS $=$ New Injury Severity Score.

fraco e de um opióide forte por um opióide fraco. Em seis casos onde a dor foi totalmente aliviada, foi utilizado analgésico opióide em quatro casos, AINH em um caso e analgésico simples em outro.

Apenas sete pacientes foram medicados na sala de emergência, após o atendimento inicial. Verificou-se que em três pacientes foram utilizados agentes opióides, três casos analgésicos simples e um caso AINH. Nenhum paciente teve sua dor aliviada totalmente e três pacientes permaneceram com a mesma intensidade dolorosa.

Verificou-se que em seis casos agentes opióides foram utilizados, nove foram utilizados analgésicos simples e dois AINH. Em apenas um caso houve alívio total da dor com a utilização de opióide (medicado duas vezes).

Um achado importante a ser citado é que dos 42 pacientes que receberam algum tipo de analgésico, 27(64,3\%) tinham sido acometidos isolada ou concomitante com outras lesões, na região corpórea de membros.

\section{DISCUSSÃO}

Os achados apresentados apontam para quatro aspectos relevantes: a confirmação da dor como um evento que acompanha o trauma, a magni-tude da intensidade dolorosa vivenciada por esses pacientes, a oligoanalgesia no setor de emergência e a falta de padronização analgésica.

A dor observada nesses pacientes, apesar de evidente no atendimento ao trauma-tizado, é pouco descrita em nosso meio, e os aspectos referentes a analgesia, muitas vezes desconsiderados. A literatura aponta a dor após o trauma 
Quadro 3. Medicações utilizadas no período de observação de pacientes vítimas de acidentes de transporte. São Paulo, maio e junho de 2002.

\begin{tabular}{|c|c|c|}
\hline $\begin{array}{c}\text { Dor I - Dor II } \\
\text { EAV }\end{array}$ & NISS & Período de observação \\
\hline $7-6$ & 9 & Tramadol - 50mg IV, dose única \\
\hline $9-9$ & 17 & Tramadol - 50mg IV, dose única \\
\hline $10-5$ & 3 & Dipirona $-2 \mathrm{ml} \mathrm{IV}$, dose única \\
\hline $8-6$ & 22 & Diclofenaco - IM, dose única \\
\hline $8-0$ & 13 & Tramadol - IV (duas doses) \\
\hline $3-3$ & 17 & Dipirona - 30 gts, dose única \\
\hline $5-5$ & 12 & Dipirona $-4 \mathrm{ml} \mathrm{IV}$, dose única \\
\hline $10-4$ & 5 & Tramadol - 50mg IV (duas doses) \\
\hline $10-7$ & 12 & Diclofenaco - IM, dose única \\
\hline $8-4$ & 27 & Dipirona - 2ml IV, dose única \\
\hline $8-4$ & 9 & $\begin{array}{l}\text { Meperidina - sol. decimal 3ml IV, dose } \\
\text { única }\end{array}$ \\
\hline $10-10$ & 5 & Tramadol - 50mg IV, dose única \\
\hline $9-6$ & 5 & Dipirona $-2 \mathrm{ml} I V$, dose única \\
\hline $5-1$ & 2 & Dipirona $-2 \mathrm{ml} \mathrm{IV}$, dose única \\
\hline $8-3$ & 3 & Dipirona $-2 \mathrm{ml} I V$, dose única \\
\hline $10-10$ & 4 & Dipirona $-2 \mathrm{ml} I V$, dose única \\
\hline $8-6$ & 11 & Dipirona $-2 \mathrm{ml} \mathrm{IV}$, dose única \\
\hline
\end{tabular}

EAV-Escala An alógica Visual; ;NISS $=$ New Injury Severity Score.

como intensa e moderada e a indicação analgésica pouco considerada, achado confirmado nesse estudo ${ }^{(8)}$.

Ao analisar-se o tipo de medicação utilizada, constatou-se que os analgésicos simples ainda aparecem com grande ênfase, praticamente empatados com os opióides e que, conforme pode se observar nos Quadros 1 a 3, a dose e o intervalo de administração dos opióides foi, muitas vezes, aquém do recomendado para adultos, com grandes intervalos entre as doses ou em doses únicas.

Diversos estudos apontam que a baixa e inconstante utilização de analgésicos opióides no setor de emergência é a principal causa da permanência da dor moderada e intensa, em vítimas de trauma e dor aguda em geral $^{(9-11)}$.

A síntese dos Quadros 1 a 3 permite afirmar que as vítimas que receberam tratamento antiálgico em dois momentos tiveram sua dor aliviada em seis casos, dos sete totais observados no estudo.

A permanência da dor ou a diminuta redução da mesma em alguns casos pode estar relacionada à indicação esporádica de medicamentos, ou apenas quando solicitados, e não de forma regular (horário) e em esquema "se necessário", o que permite uma dose denominada por estudiosos em dor como dose de "escape", quando solicitada pelo paciente ${ }^{(10)}$.

A indicação na prescrição da forma "se necessário", de modo isolado, é considerada errônea, uma vez que não estabelece horários regulares para a administração e ação do fármaco. No entanto, é aceita como eficaz para auxiliar no tratamento da dor, uma vez que permite, entre as doses regulares, que seja feita uma intervenção analgésica, em casos de dores de maior intensidade, contínuas e/ou não aliviadas com os fármacos de horário(11-12).
A utilização de opióides de forma isolada e em dose única não se mostra eficaz, e essa realidade foi encontrada nesta pesquisa. No entanto, a indicação de opióides para dor moderada e intensa mostrou resultados importantes na diminuição da dor, apesar das doses únicas ou isoladas (Quadros 1 a 3). A ação da maioria dos opióides administrados na forma intravenosa é de cinco a dez minutos, alcançando o seu pico de ação em tempo inferior a quarenta minutos $^{(12-13)}$.

Além disso, os dados da Tabela 2 mostram que a dor não melhora sozinha, mas que é necessária a administração de analgésicos para a obtenção de resultados positivos.

Um outro aspecto, que merece destaque nessa discussão, diz respeito a falta de concordância na prescrição de analgesia pelas equipes, observada pela pesquisadora em campo, ou seja, que para um mesmo tipo de lesão, fratura exposta, quatro ou cinco tipos de propostas analgésicas foram prescritas, incluindo dipirona 35 gotas via oral de $6 / 6$ horas ou se necessário até morfina intravenosa de $4 / 4$ horas.

Acreditou-se importante exemplificar casos como esse, pois a literatura reconhece esse tipo de lesão como de dor intensa, na qual o uso de opióides é indicado ${ }^{(14)}$.

Estudo nacional que analisou a preva-lência e as características da dor em 139 vítimas de acidentes de causa externa na fase pré-hospitalar, descreveu os medicamentos analgésicos existentes nas viaturas e os procedimentos de analgesia implementados. O estudo foi feito no prontosocorro do Hospital das Clínicas da FMUSP durante 12 finais de semana. Todas as vitimas de acidentes de causa externa removidas por viaturas de saúde, admitidas na sala de urgência do pronto-socorro e a equipe envolvida no resgate foram entrevistadas. Havia queixa de dor no momento do trauma em 67 (48,2\%) das vítimas, e no momento da entrevista em 87 (62,6\%) delas. Analgesia episódica ocorreu em 13,9\% dos entrevistados. A dor foi avaliada como intensa por $44 \%$ das vitimas, como moderada por $21,5 \%$ e insuportável em $19 \%$ delas. A média de intensidade de dor foi 6,5. Os locais mais freqüentes de queixa álgica foram MMII (30,6\%), MMSS $(21,9 \%)$ e crânio $(18,1 \%)$, o que correspondeu ao local da lesão. A maioria $(75,5 \%)$ dos profissionais envolvidos na remoção eram bombeiros. Havia médico em 15\% dos veículos e algum profissional de enfermagem em 21,6\%. Em cerca de 14,5\% das viaturas havia medicação analgésica, utilizada apenas em seis $(4,3 \%)$ das remoções ${ }^{(15)}$.

Um outro estudo com a finalidade de avaliar o conhecimento sobre dor aguda e seu controle entre médicos e acadêmicos do sexto ano no setor de emergência, constatam que dos entrevistados, 50\% consideraram-se em condições de tratar a dor na maioria das ocasiões, porém, expressiva proporção de internos e residentes (40,6\%) não se 
consideravam em condições de fazê-lo. Além disso, significativa parcela dos entrevistados $(52,0 \%)$ disseram prescrever analgésicos apenas quando necessário e não de forma regular. Esse fato é comum e preocupante em qualquer setor hospitalar, uma vez que, para o adequado alívio e tratamento da dor, são necessárias avaliações freqüentes, realizadas por profissional capacitado, e documentadas no prontuário por participante da equipe multiprofissional ${ }^{(16)}$.

Acredita-se que o aspecto mais importante na implementação da avaliação álgica, e posterior tratamento, seja padronizar uma conduta, ou seja, fazer com que todo profissional envolvido no atendimento a pacientes traumatizados investigue a dor, que essa prática se torne uma rotina e, a partir daí, a melhor conduta analgésica seja adotada, como ocorreu em nosso país, com as etapas do ATLS, que determina as condutas no atendimento ao trauma ${ }^{(5)}$.

Em relação ao número de vítimas que receberam analgesia, constatou-se que $42(46,7 \%)$ foram medicadas e $48(53,3 \%)$ permaneceram sem nenhum tipo de medicação antiálgica por no mínimo três horas após a avaliação inicial. Esse achado confirma, de forma contundente, o problema da oligoanalgesia no trauma.

Analisando 401 pacientes admitidos para tratamento com fraturas no setor de emergência, pesquisadoras americanas observaram que apenas 121(30,0\%) receberam algum tipo de analgesia durante um período de quatro horas ${ }^{(17)}$.

Dos 100 pacientes entrevistados na avaliação inicial, 90 $(90,0 \%)$ referiram dor. Considerando que a dor moderada e intensa ocorreu em $85,0 \%$ das vezes (Tabela 1), e que, para esta magnitude de dor, estão indicados opióides fracos e fortes, fica clara a pouca ou nenhuma melhora observada em alguns pacientes.

Outro fato a ser destacado, é a frequência de prescrição em dose única, considerando que o quadro doloroso, possivelmente, levará dias para desaparecer. A produção de substâncias algogênicas como prostaglandina e serotonina, em situações de lesões por trauma, vão diminuindo com o passar dos dias e da cicatrização da lesão tecidual ${ }^{(11-12,16)}$. Desse modo, a frequente avaliação da dor, assim como a efetividade da ação dos fármacos, deverá ser realizada como um cuidado importante da equipe de saúde, não esquecendo que a dor é

\section{REFERÊNCIAS}

1. Clarke WC. A mensuração da dor. In: Kanner R, organizador. Segredos em clínica de dor. Porto Alegre: Artmed; 1998. p. 35-50.

2. Calil AM, Pimenta CAM, Birolini D. The "oligoanalgesia problem" in the emergency care. Clinics. 2007;62(5):591-8.

3. Teixeira MJ. Fisiopatologia da dor. Rev Med (São Paulo). 1995;73(2):55-64.

4. International Association for Study of Pain (IASP). Consensus development conference statement: the integrated approach to the management of pain. Accid Emerg Med. 1994;6(3): (document number - 491-292).

5. American College of Surgeons (ACS). Committee on Trauma. Suporte Avançado de Vida no Trauma - SAVT. considerada como o quinto sinal vital ${ }^{(2)}$.

Dos 42 pacientes que foram medicados, apenas 18 receberam fármacos mais de uma vez (em dois momentos) e 24 apenas uma única vez. Esse dado é de extrema importância, pois sugere que a dor, assim como outros parâmetros vitais, deve ser avaliada com frequência, de forma padronizada, por meio de instrumentos que quantifiquem a sua intensidade e forneçam uma orientação precisa sobre a melhora ou não do quadro álgico ${ }^{(13)}$.

No caso específico da dipirona, fármaco muito prescrito, sugere uma analgesia insuficiente para a gravidade e freqüência de lesões no paciente após o trauma. Não se questiona aqui, o efeito e a importância dessa droga, mas a sua indicação isolada para dores de média e forte intensidade, pós-trauma ${ }^{(10,14)}$.

Apesar da avaliação ter sido realizada em apenas um centro de atendimento ao trauma, evidenciou-se que a dor no setor de emergência constitui-se num problema real, de proporções pouco conhecidas em nosso meio. Nenhuma padronização referente a analgesia em vítimas de trauma foi encontrada pela pesquisadora em cinco hospitais de nível terciário no Município de São Paulo visitados em outubro de 2008, assim como estudos recentes na literatura nacional que abordem essa temática,o que reforça a necesidade de novos estudos.

\section{CONCLUSÃO}

A dor foi encontrada em $90,0 \%$ da população estudada, sendo as dores de média e forte intensidade, as mais freqüentes. Permaneceram sem analgesia 48 (53,3\%) dos pacientes. Houve uma distribuição eqüitativa entre o uso de analgésicos simples e opióides, sem contudo identificar uma padronização em relação ao seu uso e dosagem.Diferenças estatísticas foram encontradas entre os pacientes que receberam e aqueles que não receberam analgesia em relação à melhora do quadro álgico. No hospital do estudo, não se utilizam instrumentos objetivos para aferir as queixas álgicas. Espera-se que esses achados sirvam de parâmetros para o desenvolvimento de protocolos de analgesia no setor de emergência, e para a utilização de escalas de dor.
Programa para Médicos. Trad. do Programa ATLS. São Paulo; 2006.

6. Organização Mundial da Saúde. Classificação estatística internacional de doenças e problemas relacionados à saúde. $10^{\mathrm{a}}$ ed. São Paulo: Centro Colaborador da OMS para Classificação de Doenças em Português; 1998. 2 v.

7. Fletcher RH, Fletcher SW. Epidemiologia clínica: elementos essenciais. 4a. ed. Porto Alegre: Artmed; 2006.

8. Kelly AM. A process approach to improving pain management in the emergency department: development and evaluation. J Accid Emerg Med. 2001;17(3):185-7.

9. McCaffery M, Pasero C. How to choose the best route for an opioid. Nursing. 2000;30(12):34-9; quiz 40. 
10. Alpen MA, Morse C. Managing the pain of traumatic injury. Crit Care Nurs Clin North Am. 2001;13(2):243-57.

11. Slaughter A, Pasero C, Manworren R. Unacceptable pain levels. Am J Nurs. 2002;102(5):75,7.

12. Gut Stein HB, Akil G. Opioid analgesic. In: Hardman JG, Limbird LE, Gilman AG, editors. Goodman \& Gilman's the pharmacological basis of therapeutics. 10th ed. New York: McGraw-Hill; c2001. p. 745-89.

13. Holford NHG. Farmacocinética e farmacodinâmica: determinação racional das doses e a escala temporal da ação dos fármacos. IN: Katsung BG, organizador. Farmacologia básica e clínica. Rio de janeiro: Guanabara
Koogan; 2003. p. 29-42.

14. Lewis LM, Lasater LC, Brooks CB. Are emergency physicians too stingy with analgesics? South Med J. 1994;87(1):7-9.

15. Pimenta CAM, Carvalho MB, Fogaça V. Dor e analgesia no atendimento pré-hospitalar às vítimas de acidente de causa externa. Âmbito Hosp. 2003;14(160):8-13.

16. Teixeira MJ, Fonoff ET, Lepski G, Marcon RM, Rocha RO. Dor no atendimento em pronto-socorro: avaliação do conhecimento da equipe de saúde sobre dor, analgesia e procedimentos prescritos para o controle. Rev Med (São Paulo). 1999;78(3):359-63. 